\title{
Discoidin Domain Receptor I, a Potential Biomarker and Therapeutic Target in Hepatocellular Carcinoma
}

\author{
Linghong Wu*, Xinhua Zhao*, Huan Ma, Lili Zhang, Xiaoan Li
}

Department of Gastroenterology, Mianyang Central Hospital, School of Medicine, University of Electronic Science and Technology of China, Mianyang, Sichuan, People's Republic of China

*These authors contributed equally to this work

Correspondence: Xiaoan Li, Department of Gastroenterology, Mianyang Central Hospital, School of Medicine, University of Electronic Science and Technology of China, No. 12 Changjia Lane, Jingzhong Street, Fucheng District, Mianyang, 62 1000, Sichuan, People’s Republic of China, Tel +86 8162243593 Email lixiaoan@uestc.edu.cn

\begin{abstract}
Hepatocellular carcinoma (HCC) is still one of the most lethal human cancers in the world due to its high degree of malignancy, easy invasion and metastasis, poor therapeutic effect and poor prognosis. Nowadays, there is no very effective diagnosis and treatment method. It is crucial to elucidate the underlying pathogenesis and mechanisms of HCC for developing new and effective diagnostic/ prognostic biomarkers and therapies. Discoidin domain receptors (DDRs) belong to the family of transmembrane receptor tyrosine kinases (RTKs) and are recognized as playing central regulatory roles in a variety of high incidence human diseases, including tumors. DDRs have two members, DDR1 and DDR2. The role of DDR1 in several tumors has been extensively studied, and many researchers have identified it as a powerful candidate target for the development of functional and effective tumor treatment inhibitors. However, its role and mechanism in HCC are ill defined. In this article, we review the advanced insights into the progression of DDR1 in HCC, particularly the ligands and mechanisms in invasion and metastasis, which may open new avenues for the therapeutic utility of HCC.
\end{abstract}

Keywords: discoidin domain receptor 1, hepatocellular carcinoma, metastasis, invasion, therapeutic target

\section{Introduction}

Hepatocellular carcinoma (HCC), the main subtype of primary liver cancer, who ranks sixth in new cases and fourth in death cases among all cancers, has become a serious human health problem worldwide. ${ }^{1}$ Many risk factors are involved in $\mathrm{HCC}$, including hepatitis B virus (HBV) and/or hepatitis C virus (HCV) infection, diabetes, obesity, aflatoxin ingestion, alcohol abuse, nonalcoholic fatty liver disease, and metabolic disease. ${ }^{2}$ While multiple advanced strategies of diagnosis and treatment, such as combining anti-angiogenic therapy and immunotherapy, ${ }^{3}$ have been applied to clinical practice in HCC, it still maintains the characteristics of high degree of malignancy, strong invasiveness, easy recurrence, metastasis and poor prognosis, which may be largely attributed to insufficient understanding of the etiology and pathogenesis. ${ }^{4,5}$ Thus, it is crucial to elucidate the underlying pathogenesis and mechanisms of HCC for laying the foundation to develop new diagnostic/prognostic biomarkers and therapeutic approaches.

Discoidin domain receptors (DDRs) belong to the family of transmembrane receptor tyrosine kinases (RTKs) and act as extracellular matrix (ECM) protein collagen receptors and manifest slow and continuous activation kinetics. DDRs are formed from two members: DDR1 and DDR2. As previously described that DDR1 is mainly expressed in epithelial cells, stimulated peripheral blood mononuclear cells, and T-cells while DDR2 is found in mesenchymal cells including fibroblasts, myofibroblasts, smooth muscle cells, and chondrocytes. ${ }^{6-8}$ Collective evidences suggest that dysregulation of DDRs are involved in human fibrosis, atherosclerosis, neurodegeneration, inflammatory disorders and even cancer. ${ }^{8}$ Among them, DDR1 has been extensively studied in a variety of tumors. Recently, some researchers and groups have disclosed the vital role of DDR1 in the pathogenesis of $\mathrm{HCC}$, and its potential as a diagnostic and therapeutic target is clearly emerging. Today, there is no review article about DDR1 in HCC. Thus, in this article, we will review the advanced insights into the progression of DDR1 in HCC, based on recent reports. 


\section{Composition and Structure of DDRI}

The DDR1 gene is found to be located on the p arm of human chromosome $6(6 \mathrm{p} 21.3),{ }^{9}$ and has five isoforms, DDR1a-e. All isoforms mainly contains three parts: extracellular binding domain, transmembrane (TM) domain, and intracellular domain. The extracellular binding domain consists of a discoidin (DS) domain and a DS-like domain, the latter of which binds to collagen. In the TM domain, there are two main components: an extracellular juxtamembrane (ECJM) region containing phosphorylable tyrosine and a TM helix mediating collagen independent receptor dimerization. Of the two, the former is the docking site of DDR binding proteins. The intracellular domain is composed of an intracellular juxtamembrane (ICJM) region and a catalytic tyrosine kinase domain that controls the activity of the intrinsic enzymatic. ${ }^{10}$ Alternative splicing causes changes in the ICJM domain or kinase domain. Concretely, DDR1b is more 37 amino acids (AA) than DDR1a (876 AA in total) in ICJM region. The kinase domain of DDR1c (919 AA) has 6 more AA based on DDR1b. DDR1d consists of 508 AA and devoid of the kinase domain, while DDR1e (767 AA) has a nonfunctional or inactive kinase domain (shown in Figure 1). ${ }^{11,12}$

\section{Functions of DDRI in HCC}

As mentioned in previous reports, DDR1 regulates key cellular processes, including cell proliferation, cell differentiation, cell survival and cell migration. ${ }^{12}$ Additionally, DDR1 can control ECM remodeling. Further studies have shown that DDR1 is highly expressed in many tumors, such as breast, ${ }^{13}$ lung, ${ }^{14}$ bladder, ${ }^{15}$ ovary, ${ }^{16}$ prostate, ${ }^{17}$ pancreas ${ }^{18}$ and brain, ${ }^{19}$ and the overexpression of DDR1 promotes tumor proliferation, migration, invasion and metastasis, resulting in poor prognosis. ${ }^{8}$ In HCC, DDR1 has been proven to be associated with recurrence, ${ }^{20}$ and its high expression leads to increased cancer cell migration, invasion and metastasis. ${ }^{21}$ Moreover, DDR1 expression is positively with the clinical grade of HCC, and negatively with 7-year survival rate of HCC. ${ }^{22}$ Detailed ligands and mechanisms of DDR1 in HCC are summarized below.

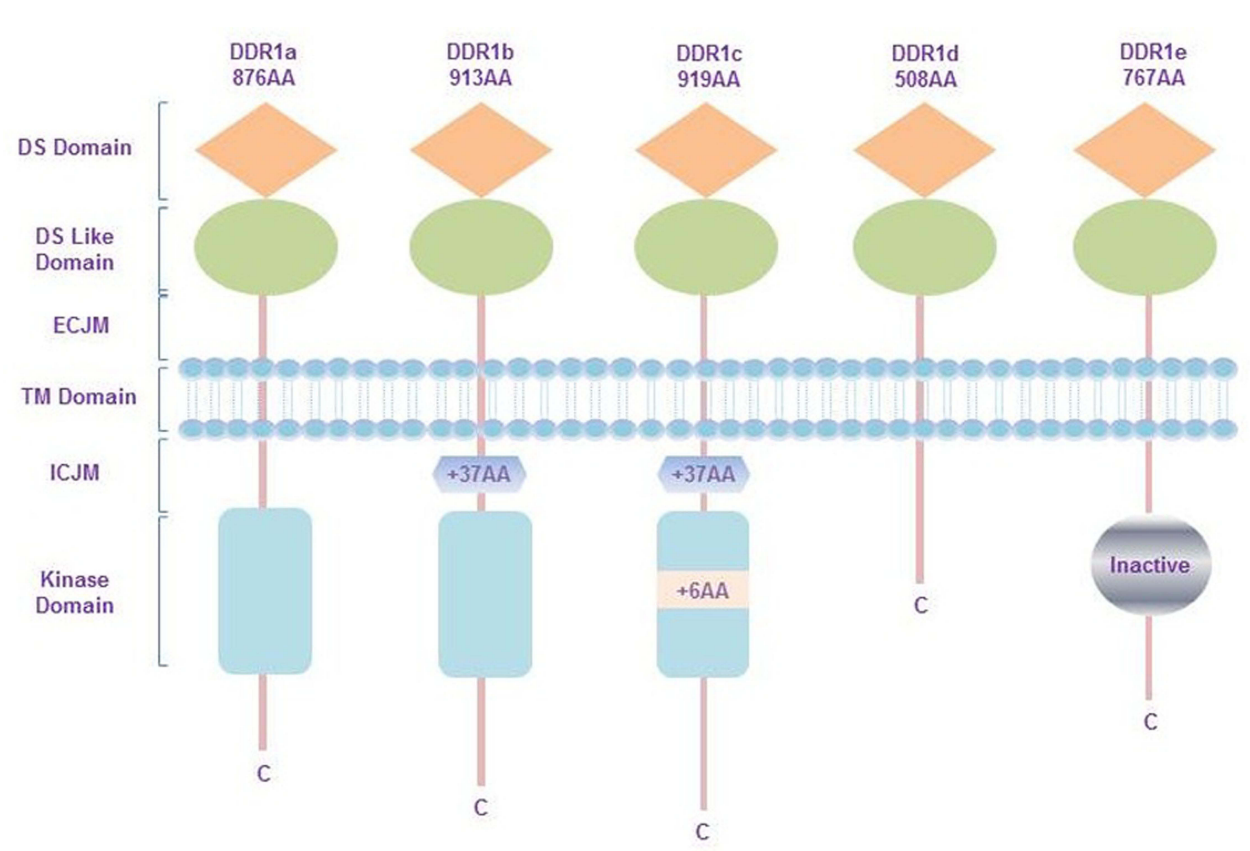

Figure I Structure and isoform of DDRI. DDR Ia contains 876 amino acids in total, and DDR Ib in ICJM region is more 37 amino acids than DDR Ia. DDR Ic has 6 more amino acids in kinase domain than DDRIb. DDRId consists of 508 amino acids and devoid of the kinase domain, while DDRle is made up of 767 AA and has a nonfunctional or inactive kinase domain.

Abbreviations: AA, amino acids; DS, discoidin; ECJM, extracellular juxtamembrane; TM, transmembrane; ICJM, intracellular juxtamembrane. 


\section{Ligands and Mechanisms of DDRI in HCC Collagen}

Collagen(s) are the most abundant ECM proteins found in mammalian tissues, and consist of fibrous collagens, networkforming collagens or other categories. Collagen is one of the ligands of DDR1. The DDR-collagen interaction is complex. In the DDR activation loop, collagen binding stimulates tyrosine kinase Src, which phosphorylates tyrosine. ${ }^{12}$ Next, activation of the kinase domain leads to auto-phosphorylation of multiple additional tyrosine in the juxtamembrane region, which then recruits downstream adaptor proteins to regulate cellular behavior, such as cell proliferation, differentiation, migration, adhesion, invasion, and metastasis. ${ }^{23}$ For example, in liver cirrhosis, DDR1a overexpression increases adhesion and reduces migration on ECM substrates. ${ }^{24}$ Indeed, DDR1 is activated by type I-V and VIII collagen, especially types I and III collagen. ${ }^{25}$ As an important component of tumor ECM, type I collagen performs high density and distorted structure in malignant cancer, which is related to tumor formation and metastasis. ${ }^{26}$ Park et al demonstrated that DDR1 is activated and tyrosine-phosphorylated by type I collagen in HCC cell lines, as well as in vitro, DDR1 overexpression stimulates HCC cell migration and invasion. ${ }^{21}$ Interestingly, an emerging view, based on compelling evidence, is that DDR1 can be activated by not only collagen binding but also interaction with the insulinlike growth factor (IIGF) system. ${ }^{27}$ Insulin, IGF1, and IGF2 are reported in a collagen-independent manner to promote DDR1 expression and phosphorylation. ${ }^{28}$ But the regulation mechanism of DDR1 and IIGF in HCC should be further studied.

\section{$\mathrm{Clq}$}

$\mathrm{C} 1 \mathrm{q}$ is the first recognized subcomponent of the classical complement pathway. When combined with $\mathrm{C} 1 \mathrm{r}$ and $\mathrm{C} 1 \mathrm{~s}$, it forms a $\mathrm{C} 1$ complex that allows the activation of the complement cascade. ${ }^{29} \mathrm{In}$ serum, about $70 \%$ of $\mathrm{C} 1$ exists as a $\mathrm{C} 1$ complex and the remaining $\mathrm{C} 1$ dissociates into $\mathrm{C} 1 \mathrm{q}$ and $\mathrm{C} 1 \mathrm{r}-\mathrm{C} 1 \mathrm{~s}$. $\mathrm{C} 1 \mathrm{q}$ is composed of three kinds of polypeptide chains, $\mathrm{A}, \mathrm{B}$, and $\mathrm{C}$. Each chain contains an N-terminal collagen-like region and a C-terminal globular head region. ${ }^{30}$ The $\mathrm{C} 1 \mathrm{q}$ 's unique structure and functional characteristics make it versatile. ${ }^{31}$ It has been reported that $\mathrm{C} 1 \mathrm{q}$ possesses multiple functions such as cell differentiation, migration, invasion, survival and adhesion, as well as complement mediated by cell surface receptors. ${ }^{32}$ Migration and invasion assays showed that $\mathrm{C} 1 \mathrm{q}$ significantly increased the average numbers of migrating and invading HepG2 cells. ${ }^{33}$ Lee et $\mathrm{al}^{33}$ proposed that $\mathrm{C} 1 \mathrm{q}$ is a novel ligand, which bound directly to DDR1 because of possessing collagen-like portion. They also examined the levels of mitogen activated protein kinases (MAPKs) proteins, phosphoinositide 3-kinase (PI3K) and Akt in HepG2 cells treated with or without C1q, and found that C1q induced the activations of MAPKs and PI3K/Akt signaling. When DDR1 is knocked down, C1q-induced migration and invasion are inhibited, and the expressions of MMP-2 and -9 are significantly reduced. Overall, it revealed that complement C1q stimulates HCC invasion and metastasis by activating DDR1.

\section{MMP-2 and MMP-9}

ECM protein degradation is an indispensable step in tumor invasion and metastasis, which is mediated by various proteases. Matrix metalloproteinase (MMP) are zinc-dependent endopeptidase that can degrade the ECM components and play key roles in the progression and dissemination of cancer. ${ }^{34}$ Multiple lines of evidences point to an important role for MMP-2 and -9 in HCC invasion and metastasis. ${ }^{35,36}$ Park et al ${ }^{21}$ added collagen type I to stimulate Huh-7 and HLE cell lines in culture medium, and found that collagen type I activates and tyrosine-phosphorylates DDR1. DDR1 overexpression increased migration and invasion in HCC cells. Then they used Zymography to assess the association between the invasion of DDR1 overexpressing cells and the activation of MMP-2 and -9. The data showed that MMP-2 and -9 are highly expressed in the DDR1 overexpressed HCC cells. What's more, in overexpressed DDR1 cells, MMP-2 is more active in HLE than in Huh-7. These findings reveal that the enhanced migration and invasion ability of HCC cells overexpressing DDR1 is mediated by MMP, especially the activated MMP-2 in HLE cells. 


\section{TGF- $\beta$ I}

Transforming growth factor- $\beta 1$ (TGF- $\beta 1$ ) is secreted by hepatic stellate cells, myofibroblasts and cancer-associated fibroblast, is an important player in HCC development and progression. ${ }^{37,38}$ It regulates many cellular pathways and participates in epithelial-mesenchymal transition to promote HCC cell migratory ability. ${ }^{39}$ At the cellular level, TGF- $\beta 1$ is described to enhance invadosome formation, maturation and activity in different cellular models. ${ }^{40}$ Invadosomes are highly complex molecular structures that couple actin polymerization with proteolytic activity to orchestrate extracellular matrix remodeling and invasion processes. ${ }^{41}$ Juin et al identified the linear invadosomes can be induced by the fibrillar type I collagen, ${ }^{42}$ and their formation is dependent on DDR $1 .{ }^{43}$ Therefore, based on these data, Zakaria Ezzoukhry and his team ${ }^{44}$ tested the impact of TGF- $\beta 1$ on DDR1, which is involved in linear invadosome formation. The results revealed that TGF- $\beta 1$ promotes linear invadosome formation in HCC cells, through DDR1 up-regulation and collagen I cross-linking.

\section{STAT3}

Signal transducer and activator of transcription 3 (STAT3) is one of seven STAT family members involved with the regulation of cellular growth, differentiation and survival. It is located in the cytoplasm, and when activated, it can be transferred into the nucleus to bind to DNA. ${ }^{45}$ Studies have shown that STAT3 has dual functions, including signal transduction and transcriptional regulation, ${ }^{46}$ and can enter the nucleus to regulate various genes expression after been activated. ${ }^{47}$ However, the relationship of STAT3 and DDR1 in HCC is still unknown. Lin et al ${ }^{22}$ confirmed that DDR1 overexpression increased STAT3 expression and promoted STAT3 phosphorylation in HCC. In turn, STAT3 overexpression increased the expression of DDR1. DDR1 and STAT3 co-immunoprecipitated mutually, and STAT3 could bind to the DDR1 promoters in nucleus. Further experiments showed that the silencing of DDR1 or STAT3 mutually eliminated the promotion of cell proliferation, migration, invasion and metastasis. Moreover, they evaluated the effect of DDR1 on the metabolism of glutamine ${ }^{22}$, which is a crucial nutrient that provides carbon and nitrogen sources and plays an important role in cancer. They found that the upregulation of DDR1 increased the expression of GLUD1 (glutamate dehydrogenase 1), GLS1 (glutaminase) and SLC1A (glutamate transporter), while si-DDR1 decreased the expression of GLUD1, GLS1 and SLC1A. All these results indicate that DDR1 and STAT3 interact to form a positive feedback pathway and synergistically promote glutamine metabolism and tumor progression in HCC.

\section{EMT}

Epithelial-mesenchymal transition (EMT) is a cellular program that converts resting epithelial cells into active mesenchymal cells, thus promotes the invasion and metastasis of tumor cells. Tumor cells with EMT express fewer epithelial markers, including E-cadherin and cytokeratins, but more mesenchymal markers, including Vimentin and N-cadherin. ${ }^{48}$ EMT is also critical for epithelial cancer cells to acquire a malignant phenotype. ${ }^{49}$ In the study, ${ }^{33} \mathrm{Clq}$ increased the expression of N-cadherin and Vimentin in HCC cells and decreased that of E-cadherin, while C1q showed the opposite data after DDR1 was knocked down, which suggest that C1q regulates EMT via DDR1. Another study ${ }^{22}$ investigated the relationship of EMT related genes expression and DDR1/STAT3, results showed that DDR1 increased the expression of Vimentin and N-cadherin in HCC cells and decreased that of E-cadherin, while siSTAT3 showd contrary results in DDR1transtected cells, which indicate that DDR1 promotes EMT via STAT3 in HCC. These mechanisms (Figure 2) provide the basis for new targeted therapies for HCC.

\section{Inhibitors of DDR I}

DDR1 is a crucial player for cancer, notably in invasion and metastasis formation. In many cancers, Overexpression of DDR1 is associated with poor overall survival. Therefore, DDR may be a potential biomarker and therapeutic target for cancer, so much so that research on DDR inhibitor is booming. Due to the structural similarity of the kinase domain, DDR1 can be targeted by tyrosine kinase receptor such as dasatinib, imatinib, nilotinib and ponatinib. ${ }^{50,51}$ To be specific, dasatinib inhibits the migration and invasion by inhibiting DDR1 expression in gastric cancer cells, ${ }^{52}$ nilotinib reduces metastatic colorectal cancer invasion through inhibiting DDR1 kinase activation. ${ }^{53}$ The disadvantage 




Figure 2 Schematic diagram of ligand and mechanism of DDRI in HCC. DDRI is positively promoting HCC progression through multiple pathways. Different colored arrows represent different pathway. Among these mechanisms, DDRI and STAT3 form a feedback loop.

Abbreviations: TGF- $\beta$ I, transforming growth factor- $\beta$ I; MAPK, mitogen activated protein kinase; PI3K/AKT, phosphatidylinositol-3-kinase/protein kinase B; STAT3, signal transducer and activator of transcription 3; MMP, matrix metalloproteinase; EMT, epithelial-mesenchymal transition.

of these inhibitors is that they are multi-targeted kinase inhibitors that target both DDRs and other receptor tyrosine kinases. Through the efforts of researchers, selective DDR1 kinase inhibitors have been generated in recent years. 48B3, a DDR1 monoclonal antibody, is reported to reduce invasion and adhesion mediated by DDR1a in glioma cell. ${ }^{54}$ T4H11-DM4, an antibody-drug conjugate targeting DDR1 which carries the tubulin inhibitor payload DM4, is demonstrated potent antitumor efficacy for colon cancer both in vitro and in vivo. ${ }^{55}$ Moreover, a large body of emerging evidences have shown that DDR1 can be targeted specifically by DDR1-IN-1 or DDR1-IN-2, ${ }^{56}$ pyrazolopyramidine derivatives $\left(7 \mathrm{rh}\right.$ and $7 \mathrm{rj}$ ), ${ }^{57}$ benzamide derivatives (compounds 23 and 24$),{ }^{58}$ tetrahydroisoquinoline derivatives (compound 10), ${ }^{59} 3^{\prime}$-(imidazo[1,2-A] pyrazin-3-yl)-[1,1'-biphenyl]-3-carboxamide (compound $\left.8 \mathrm{v}\right)^{60}$ and so on. These inhibitors have a certain positive effects in the treatment of some tumors. Unfortunately, there are no reports on the treatment of HCC with DDR1 inhibitors.

\section{Conclusions and Future Perspectives}

HCC is among the most frequent causes of cancer-related death worldwide, and there is currently no most available and effective treatment strategy. DDR1 is emerging as an attractive new tumor biomarker and therapeutic target, given the key role that it plays in the progression of HCC. Now, a variety of DDR1 inhibitors with different mechanisms of action have been investigated, and they have the potential to treat human cancers, ${ }^{61}$ thereby helping to improve the level of diagnosis and treatment and improve the prognosis of patients. However, they still lack specificity and none of them have been used in the treatment of HCC. Therefore, the search for more specific DDR1 inhibitors is still needed. We look 
forward to describing the role of DDR1 in detail and the entry of DDR1-specific inhibitors into HCC clinical trial to achieve encouraging cancer treatment outcomes.

\section{Disclosure}

The authors declare that they have no competing interests.

\section{References}

1. Ferlay J, Colombet M, Soerjomataram I, et al. Estimating the global cancer incidence and mortality in 2018: GLOBOCAN sources and methods. Int J Cancer. 2019;144(8):1941-1953. doi:10.1002/ijc.31937

2. Chen E, Xu X, Liu R, et al. Small but heavy role: microRNAs in hepatocellular carcinoma progression. Biomed Res Int. 2018;2018:6784607. doi:10.1155/2018/6784607

3. Solimando AG, Summa S, Vacca A, et al. Cancer-associated angiogenesis: the endothelial cell as a checkpoint for immunological patrolling. Cancers. 2020;12(11):3380. doi:10.3390/cancers12113380

4. Bruix J, Llovet JM. Major achievements in hepatocellular carcinoma. Lancet. 2009;373(9664):614-616. doi:10.1016/S0140-6736(09)60381-0

5. Sene WR, Zhang Y, Chen Y. Hepatocellular carcinoma: focus on different aspects of management. ISRN Oncol. 2012;2012:421673. doi:10.5402/ 2012/421673

6. Gadiya M, Chakraborty G. Signaling by discoidin domain receptor 1 in cancer metastasis. Cell Adh Migr. 2018;12(4):315-323. doi:10.1080/ 19336918.2018.1520556

7. Elkamhawy A, Lu Q, Nada H, et al. The journey of DDR1 and DDR2 kinase inhibitors as rising Stars in the fight against cancer. Int J Mol Sci. 2021;22:6535. doi:10.3390/ijms22126535

8. Mehta V, Chander H, Munshi A. Complex roles of discoidin domain receptor tyrosine kinases in cancer. Clin Transl Oncol. 2021;23(8):1497-1510. doi:10.1007/s12094-021-02552-6

9. Edelhoff S, Sweetser DA, Disteche CM. Mapping of the NEP receptor tyrosine kinase gene to human chromosome 6p21.3 and mouse chromosome 17C. Genomics. 1995;25(1):309-311. doi:10.1016/0888-7543(95)80144-B

10. Gao Y, Zhou J, Li J. Discoidin domain receptors orchestrate cancer progression: a focus on cancer therapies. Cancer Sci. 2021;112(3):962-969. doi:10.1111/cas. 14789

11. Abdulhussein R, Koo DHH, Vogel WF. Identification of disulfidelinked dimers of the receptor tyrosine kinase DDR1. J Biol Chem. 2008;283 (18):12026-12033. doi:10.1074/jbc.M704592200

12. Leitinger B. Discoidin domain receptor functions in physiological and pathological conditions. Int Rev Cell Mol Biol. $2014 ; 310: 39-87$. doi:10.1016/B978-0-12-800180-6.00002-5

13. Vella V, Malaguarnera R, Nicolosi ML, et al. Discoidin domain receptor 1 modulates insulin receptor signaling and biological responses in breast cancer cells. Oncotarget. 2017;8(26):43248-43270. doi:10.18632/oncotarget.18020

14. Miao L, Zhu S, Wang Y, et al. Discoidin domain receptor 1 is associated with poor prognosis of non-small cell lung cancer and promotes cell invasion via epithelial-to-mesenchymal transition. Med Oncol. 2013;30(3):626. doi:10.1007/s12032-013-0626-4

15. Xie X, He H, Zhang N, et al. Overexpression of DDR1 promotes migration, invasion, though EMT-related molecule expression and COL4A1/ DDR1/MMP-2 signaling axis. Technol Cancer Res Treat. 2020;19:1533033820973277. doi:10.1177/1533033820973277

16. Quan J, Yahata T, Adachi S, et al. Identification of receptor tyrosine kinase, discoidin domain receptor 1 (DDR1), as a potential biomarker for serous ovarian cancer. Int J Mol Sci. 2011;12(2):971-982. doi:10.3390/ijms12020971

17. Shimada K, Nakamura M, Ishida E, et al. Prostate cancer antigen-1 contributes to cell survival and invasion though discoidin receptor 1 in human prostate cancer. Cancer Sci. 2007;99(1):39-45. doi:10.1111/j.1349-7006.2007.00655.x

18. Aguilera KY, Huang $\mathrm{H}, \mathrm{Du} \mathrm{W}$, et al. Inhibition of discoidin domain receptor 1 reduces collagen-mediated tumorigenicity in pancreatic ductal adenocarcinoma. Mol Cancer Ther. 2017;16(11):2473-2485. doi:10.1158/1535-7163.MCT-16-0834

19. Ma YS, Wu ZJ, Bai RZ, et al. DRR1 promotes glioblastoma cell invasion and epithelial-mesenchymal transition via regulating AKT activation. Cancer Lett. 2018;423:86-94. doi:10.1016/j.canlet.2018.03.015

20. Jian ZX, Sun J, Chen W, et al. Involvement of discoidin domain 1 receptor in recurrence of hepatocellular carcinoma by genome-wide analysis. Med Oncol. 2012;29(5):3077-3082. doi:10.1007/s12032-012-0277-x

21. Park HS, Kim KR, Lee HJ, et al. Overexpression of discoidin domain receptor 1 increases the migration and invasion of hepatocellular carcinoma cells in association with matrix metalloproteinase. Oncol Rep. 2007;18(6):1435-1441.

22. Lin Y, Jin H, Wu X, et al. The cross-talk between DDR1 and STAT3 promotes the development of hepatocellular carcinoma. Aging. 2020;12 (14):14391-14405. doi:10.18632/aging.103482

23. Carafoli F, Hohenester E. Collagen recognition and transmembrane signalling by discoidin domain receptors. Biochim Biophys Acta. 2013;1834 (10):2187-2194. doi:10.1016/j.bbapap.2012.10.014

24. Song S, Shackel NA, Wang XM, et al. Discoidin domain receptor 1: isoform expression and potential functions in cirrhotic human liver. Am J Pathol. 2011;178(3):1134-1144. doi:10.1016/j.ajpath.2010.11.068

25. Vogel W, Gish GD, Alves F, et al. The discoidin domain receptor tyrosine kinases are activated by collagen. Mol Cell. 1997;1(1):13-23. doi:10.1016/S1097-2765(00)80003-9

26. Castro-Sanchez L, Soto-Guzman A, Guaderrama-Diaz M, et al. Role of DDR1 in the gelatinases secretion induced by native type IV collagen in MDA-MB-231 breast cancer cells. Clin Exp Metastas. 2011;28(5):463-477. doi:10.1007/s10585-011-9385-9

27. Fridman R, Agarwal G. New concepts on the interactions of discoidin domain receptors with collagen. Biochim Biophys Acta Mol Cell Res. 2019;1866(11):118527. doi:10.1016/j.bbamcr.2019.118527

28. Vella V, Malaguarnera R. The emerging role of insulin receptor isoforms in thyroid cancer: clinical implications and new perspectives. Int $J$ Mol Sci. 2018;19(12):3814. doi:10.3390/ijms 19123814 
29. Lu J, Kishore U. C1 complex: an adaptable proteolytic module for complement and non-complement functions. Front Immunol. 2017;8:592. doi:10.3389/fimmu.2017.00592

30. Ghebrehiwet B, Kandov E, Kishore U, et al. Is the A-chain the engine that drives the diversity of C1q functions? revisiting its unique structure. Front Immunol. 2018;9:162. doi:10.3389/fimmu.2018.00162

31. Kouser L, Madhukaran SP, Shastri A, et al. Emerging and novel functions of complement protein C1q. Front Immunol. 2015;6:317. doi: 10.3389/ fimmu.2015.00317

32. Nayak A, Pednekar L, Reid KBM, et al. Complement and non-complement activating functions of C1q: a prototypical innate immune molecule. Innate Immun. 2012;18(2):350-363. doi:10.1177/1753425910396252

33. Lee JH, Poudel B, Ki HH, et al. Complement C1q stimulates the progression of hepatocellular tumor through the activation of discoidin domain receptor 1. Sci Rep. 2018;8(1):4908. doi:10.1038/s41598-018-23240-6

34. Yamamoto K, Murphy G, Troeberg L. Extracellular regulation of metalloproteinases. Matrix Biol. 2015;44-46:255-263. doi:10.1016/j. matbio.2015.02.007

35. Tang Y, Lv P, Sun Z, et al. 14-3-3 $\beta$ promotes migration and invasion of human hepatocellular carcinoma cells by modulating expression of MMP2 and MMP9 through PI3K/Akt/NF-אB pathway. PLoS One. 2016;11(1):e0146070. doi:10.1371/journal.pone.0146070

36. Liu D, Kang H, Gao M, et al. Exosome-transmitted circ-MMP2 promotes hepatocellular carcinoma metasis by upregulating MMP2. Mol Oncol. 2020;14(6):1365-1380. doi:10.1002/1878-0261.12637

37. Dooley S, Dijke P. TGF-beta in progression of liver disease. Cell Tissue Res. 2012;347(1):245-256. doi:10.1007/s00441-011-1246-y

38. Neuzillet C, de Gramont A, Tijeras-Raballand A, et al. Perspectives of TGF-beta inhibition in pancreatic and hepatocellular carcinomas. Oncotarget. 2014;5(1):78-94. doi:10.18632/oncotarget.1569

39. Shrestha R, Bridle KR, Crawford DHG, et al. Immune checkpoint molecules are regulated by transforming growth factor (TGF)- $\beta 1$-induced epithelial-to-mesenchymal transition in hepatocellular carcinoma. Int J Med Sci. 2021;18:2466-2497. doi:10.7150/ijms.54239

40. Pignatelli J, Tumbarello DA, Schmidt RP, et al. Hic-5 promotes invadopodia formation and invasion during TGF-beta-induced epithelial-mesenchymal transition. J Cell Biol. 2012;197(3):421-437. doi:10.1083/jcb.201108143

41. Paterson EK, Courtneidge SA. Invadosomes are coming: new insights into function and disease relevance. FEBS J. 2018;285(1):8-27. doi:10.1111/ febs. 14123

42. Juin A, Billottet C, Moreau V, et al. Physiological type I collagen organization induces theformation of a novel class of linear invadosomes. Mol Biol Cell. 2012;23(2):297-309. doi:10.1091/mbc.e11-07-0594

43. Juin A, Martino JD, Leitinger B, et al. Discoidin domain receptor 1 controls linear invadosome formation via a Cdc42-Tuba pathway. J Cell Biol. 2014;207(4):517-533. doi:10.1083/jcb.201404079

44. Ezzoukhry Z, Henriet E, Piquet L, et al. TGF- $\beta 1$ promotes linear invadosome formation in hepatocellular carcinoma cells, through DDR1 upregulation and collagen I cross-linking. Eur J Cell Biol. 2016;95(11):503-512. doi:10.1016/j.ejcb.2016.09.003

45. Li R, Huang Y, Lin J. Distinct effects of general anesthetics on lung metastasis mediated by IL-6/JAK/STAT3 pathway in mouse models. Nat Commun . 2020;11(1):642. doi:10.1038/s41467-019-14065-6

46. Yu H, Pardoll D, Jove R. STATs in cancer inflammation and immunity: a leading role for STAT3. Nat Rev Cancer. 2009;9(11):798-809. doi:10.1038/nrc2734

47. Chiocca EA, Yu JS, Lukas RV, et al. Regulatable interleukin-12 gene therapy in patients with recurrent high-grade glioma: results of a Phase 1 trial. Sci Transl Med. 2019;11(505):eaaw5680. doi:10.1126/scitranslmed.aaw5680

48. Jing H, Song J, Zheng J. Discoidin domain receptor 1: new star in cancer-targeted therapy and its complex role in breast carcinoma. Oncol Lett. 2018;15(3):3403-3408. doi:10.3892/ol.2018.7795

49. Yang J, Weinberg RA. Epithelial-mesenchymal transition: at the crossroads of development and tumor metastasis. Dev Cell. 2008;14(6):818-829. doi:10.1016/j.devcel.2008.05.009

50. Day E, Waters B, Spiegel K, et al. Inhibition of collagen-induced discoidin domain receptor 1 and 2 activation by imatinib, nilotinib and dasatinib. Eur J Pharmacol. 2008;599(1-3):44-53. doi:10.1016/j.ejphar.2008.10.014

51. Canning P, Tan L, Chu K, et al. Structural mechanisms determining inhibition of the collagen receptor DDR1 by selective and multi-targeted type II kinase inhibitors. J Mol Biol. 2014;426(13):2457-2470. doi:10.1016/j.jmb.2014.04.014

52. Montenegro RC, Howarth A, Ceroni A, et al. Identification of molecular targets for the targeted treatment of gastric cancer using dasatinib. Oncotarget. 2020;11(5):535-549. doi:10.18632/oncotarget.27462

53. Jeitany M, Leroy C, Tosti P, et al. Inhibition of DDR1-BCR signalling by nilotinib as a new therapeutic strategy for metastatic colorectal cancer. EMBO Mol Med. 2018;10:e7918. doi:10.15252/emmm.201707918

54. Rosalyn R, Gustavo L, Karoly N, et al. Discoidin domain receptor-1a (DDR1a) promotes glioma cell invasion and adhesion in association with matrix metalloproteinase-2. J Neurooncol. 2006;76(3):239-248. doi:10.1007/s11060-005-6874-1

55. Tao Y, Wang R, Lai Q, et al. Targeting of DDR1 with antibody-drug conjugates has antitumor effects in a mouse model of colon carcinoma. Mol Oncol. 2019;13(9):1855-1873. doi:10.1002/1878-0261.12520

56. Kim HG, Tan L, Weisberg EL, et al. Discovery of a potent and selective DDR1 receptor tyrosine kinase inhibitor. ACS Chem Biol. 2013;8 (10):2145-2150. doi:10.1021/cb400430t

57. Kothiwale S, Borza CM, Lowe EWJ, et al. Discoidin domain receptor 1 (DDR1) kinase as target for structure-based drug discovery. Drug Discov Today. 2015;20(2):255-261. doi:10.1016/j.drudis.2014.09.025

58. Li Y, Lu X, Ren X, et al. Small molecule discoidin domain receptor kinase inhibitors and potential medical applications. $J$ Med Chem. 2015;58 (8):3287-3301. doi:10.1021/jm5012319

59. Wang Z, Bian H, Bartual SG, et al. Structure-based design of tetrahydroisoquinoline-7-carboxamides as selective Discoidin Domain Receptor 1 (DDR1) inhibitors. J Med Chem. 2016;59(12):5911-5916. doi:10.1021/acs.jmedchem.6b00140 
60. Mo C, Zhang Z, Li Y, et al. Design and optimization of 3'-(Imidazo[1,2- A]pyrazin-3-yl)-[1,1'-biphenyl]-3-carboxamides as selective DDR1 inhibitors. ACS Med Chem Lett. 2020;11:379-384. doi:10.1021/acsmedchemlett.9b00495

61. Zhavoronkov A, Ivanenkov YA, Aliper A, et al. Deep learning enables rapid identification of potent DDR1 kinase inhibitors. Nat Biotechnol. 2019;37(9):1038-1040. doi:10.1038/s41587-019-0224-x

\section{Publish your work in this journal}

The International Journal of General Medicine is an international, peer-reviewed open-access journal that focuses on general and internal medicine, pathogenesis, epidemiology, diagnosis, monitoring and treatment protocols. The journal is characterized by the rapid reporting of reviews, original research and clinical studies across all disease areas. The manuscript management system is completely online and includes a very quick and fair peer-review system, which is all easy to use. Visit http://www.dovepress.com/testimonials.php to read real quotes from published authors.

Submit your manuscript here: https://www.dovepress.com/international-journal-of-general-medicine-journal 